\title{
A Memory Grouping Method for Sharing Memory BIST Logic
}

\author{
Masahide Miyazaki, Tomokazu Yoneda, and Hideo Fujiwara \\ Graduate School of Information Science, Nara Institute of Science and Technology (NAIST), \\ 8916-5 Takayama, Ikoma, Nara 630-0101, Japan \\ Email:\{masah-mi, Yoneda, fujiwara\}@is.naist.jp
}

\begin{abstract}
With the increasing demand for SoCs to include rich functionality, SoCs are being designed with hundreds of small memories with different sizes and frequencies. If memory BIST logics were individually added to these various memories, the area overhead would be very high. To reduce the overhead, memory BIST logic must therefore be shared. This paper proposes a memory-grouping method for memory BIST logic sharing. A memory-grouping problem is formulated and an algorithm to solve the problem is proposed. Experimental results showed that the proposed method reduced the area of the memory BIST wrapper by up to $40.55 \%$. The results also showed that the ability to select from two types of connection methods produced a greater reduction in area than using a single connection method.
\end{abstract}

\section{Introduction}

With the increasing number of functions being included in SoCs, many memories with different sizes and frequencies are being used. The latest SoCs contain hundreds of memories. Testing all the memories in these SoCs sequentially would take a long time. Therefore, a memory BIST design that allows two or more memories to be tested simultaneously is needed. However, due to power-consumption constraints, not all memories can be activated at the same time. To solve this problem, a scheduling technique for minimizing the test application time under power-consumption constraints is needed. Adding individual circuits for memory BISTs to lots of small memories would result in huge area overheads. To reduce these overheads, memory BIST logic must be able to be shared.

A BIST architecture, based on a single micro-programmable BIST processor and a set of memory wrappers, was proposed to simplify the testing of systems containing many distributed SRAMs of different sizes [1]. To reduce the BIST area overhead, it was proposed to share a single wrapper between a cluster of SRAMs (same type, width, and addressing space). However, in some cases, memories that have different widths or addressing spaces can be connected and share BIST logic. There can also be two or more connection methods. To achieve a satisfactory solution, the memory-connection type should be considered along with decisions on memory groups.

In this paper, we propose two types of memory-connection methods for BIST wrapper sharing. A memory-grouping problem for test circuit minimization under constraints of power consumption and test application time is also formulated together with an algorithm that solves the problem. In addition, the effectiveness of this technique is demonstrated experimentally. This paper is organized as follows. In section II, our method for memory BIST logic sharing is described. In section III, the memory-grouping problem and an algorithm to solve the problem are presented. The experimental results are shown in section IV.

\section{Memory BIST Logic sharing}

In this section, we describe our method of BIST logic sharing for single port and word access memory. Figure 1 shows an example of a memory BIST wrapper. The data generator generates input test sequences. The address generator generates read and write addresses and the response analyzer captures test output responses and detects faults. The by-pass FFs are not used to test memory, but are used to care the memory interface signal during a scan test. The area of the address generator, data generator, and response analyzer are almost proportional to the bit width of the address, input data, and output data, respectively. However, some of these logics can be shared by different memories wherever the number of words or the data bit width are the same; hence, the area of test circuits can be reduced. In this paper, we treat the following two memory connection methods for memory BIST logic sharing: parallel connection and serial connection. Parallel connection can be used to connect memories that have the same number of words. Figure 2 shows an example of parallel connection.

In this example, three data and address generators are reduced to one by distributing the same test data and address signals from a couple of data and address generators to (1) (4), enabling four memories to be tested simultaneously. 


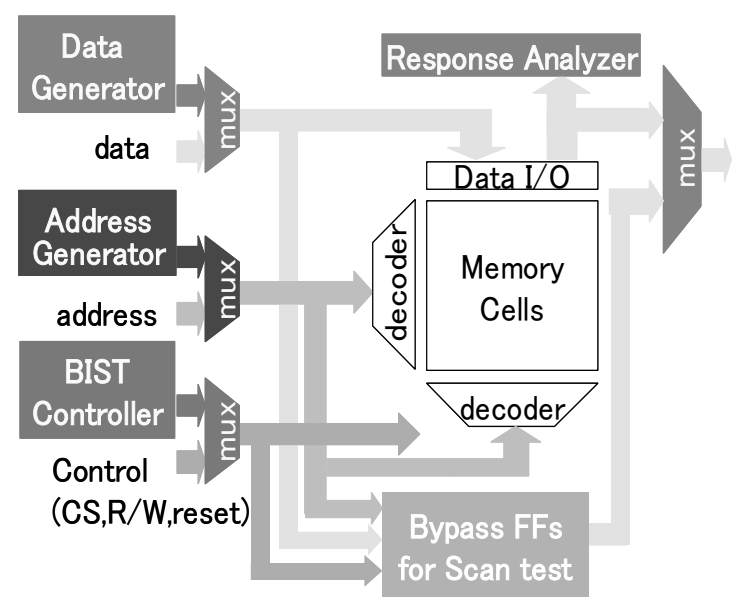

Fig.1 Memory BIST Wrapper

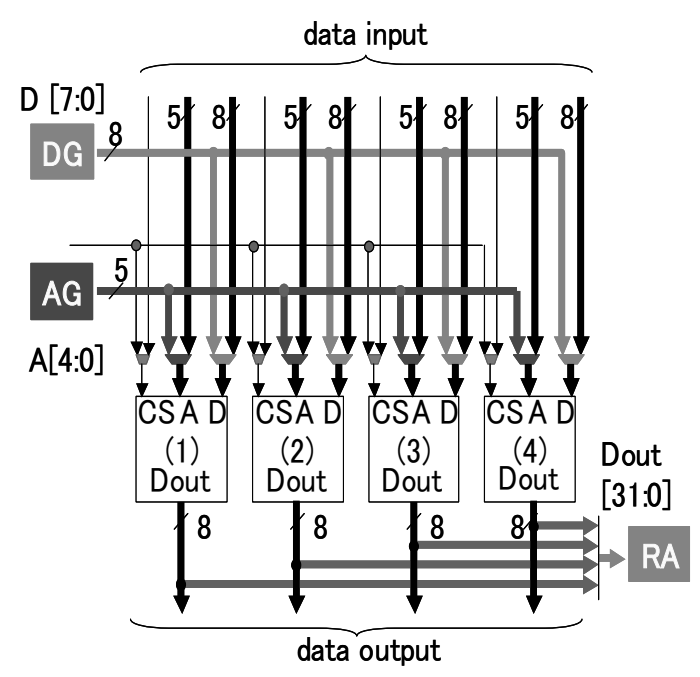

Fig.2 Parallel connection of memories

Serial connection allows memories with the same bit width to be connected. Figure 3 shows an example of four serially connected $8 \times 32$ word memories. In this example, the four memories are tested as an $8 \times 128$ word memory. The address generator generates an additional 2 bit signal, and the signal is used to select the memories from (1) - (4), enabling the four memories to be tested serially. If all the memories have individual BIST logic, a 32-bit data generator and response analyzer are required, but in this example, all the memories can be tested using a shared 8bit generator and 8 bit response analyzer.

Serial connection reduces the area more than parallel connection and also uses less power than parallel connection. However, the time required for serial connection testing is longer than that for parallel connection testing. To achieve the minimum area and a reasonable test application time under power consumption constraints, the type of memory connection should be considered during decisions on memory grouping. The layout design must also take into account distance constraints in relation to these connections.

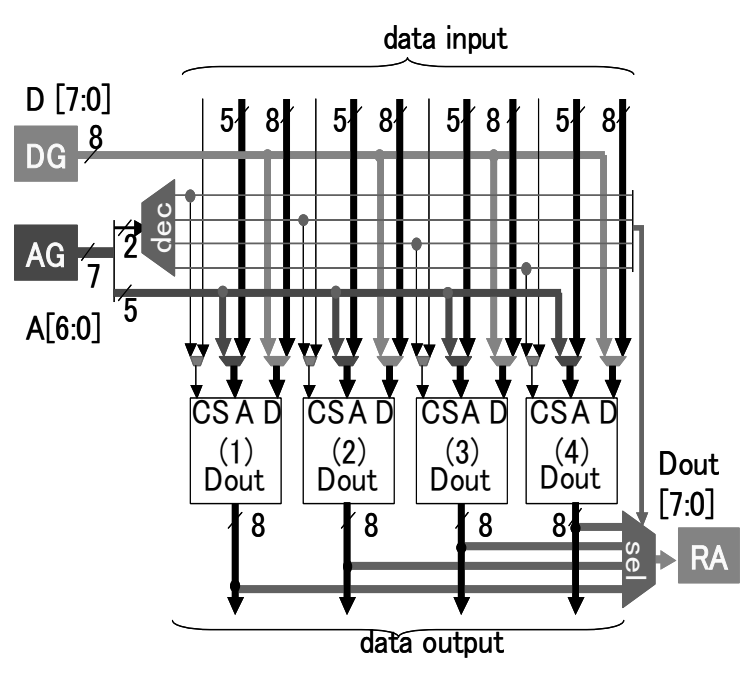

Fig.3 Serial connection of memories

\section{Memory-Grouping Problem and Algorithm}

\section{A. Formulation of Memory-Grouping Problem}

In this subsection, we present a memory-grouping problem. We assume that the following information for each memory mi is given:

- $b_{i}$ : data bit width of $m_{i}$

$-w_{i}$ : word depth of $m_{i}$

- $p_{i}$ : maximum power consumption of testing $m_{i}$

$-f_{i}$ : operating frequency of $m_{i}$

$-x_{i}$ : X coordinate of $m_{i}, y_{i}$ : Y coordinate of $m_{i}$

We define two types of compatibility, namely p-compatibility and s-compatibility, as follows:

Given a set of memories $V=\left\{m_{1}, m_{2}, \ldots m_{n}\right\}$, a pair of memories $m_{i}, m_{j} \in V$ is p-compatible if they satisfy the following conditions:

$$
\begin{aligned}
& w_{i}=w_{j} \\
& f_{i}=f_{j} \\
& \sqrt{\left(x_{i}-x_{j}\right)^{2}+\left(y_{i}-y_{j}\right)^{2}}<D
\end{aligned}
$$

$D$ is a constraint value that the designer decides according to the design condition.

P-compatibility is represented by a graph $G_{p}=\left(V, E_{p}\right)$, where $V$ is a set of a memory and the edge between a pair of vertices $\left(m_{i}, m_{j}\right) \in E_{p}$ exists if $m_{i}$ and $m_{j}$ are p-compatible. If a set of memories can be connected in parallel, the graph induced on $G_{p}$ by the memories has to be a clique.

In the same way, a pair of memories $m_{i}, m_{j} \in V$ is s-compatible if they satisfy the following conditions:

$$
\begin{aligned}
& b_{i}=b_{j} \\
& f_{i}=f_{j}
\end{aligned}
$$




$$
\sqrt{\left(x_{i}-x_{j}\right)^{2}+\left(y_{i}-y_{j}\right)^{2}}<D
$$

$\mathrm{S}$-compatibility is represented by a graph $G_{s}=\left(V, E_{s}\right)$, where $V$ is a set of memories and the edge between a pair of vertices $\left(m_{i}, m_{j}\right), \in \mathrm{E}_{\mathrm{s}}$ exists if $m_{i}$ and $m_{j}$ are s-compatible. If a set of memories can be connected serially, the graph induced on $G_{s}$ by the memories has to be a clique.

To design memory BIST wrappers using these techniques for memory BIST logic sharing, we have to find a partition of $V$ such that the memories that share the wrapper are included in the same block. Moreover, the partition $\pi=\left\{B_{1}, B_{2}, \ldots B_{k}\right\}$ has to satisfy the following conditions:

$G_{i p}$ is the graph induced on $G_{p}$ by block $B_{i}$.

$G_{i s}$ is the graph induced on $G_{s}$ by block $B_{i}$.

$G_{i p}$ or $G_{i s}$ is a clique.

When only the graph $G_{i p}\left(G_{i s}\right)$ is a clique, the memories included in $B_{i}$ are connected in parallel (serially). If $G_{i p}$ and $G_{i s}$ are both clique, we have to select the type of connection.

For a partition $\pi$, we can calculate the area of the BIST wrapper, test application time, and power consumption of each block. The area and test application time depend on the test-pattern algorithm. In this work, these were calculated according to a published design [4] using an $8 \mathrm{~N}$ algorithm as follows.

If the connection type of block $B_{i}=\left\{m_{1}, m_{2}, \ldots m_{k}\right\}$ is a parallel connection,

$$
\begin{aligned}
& \text { Area } S_{B i}=0.75\left(\log _{2}\left(w_{B i}\right)\right)^{2}+2 k \log _{2}\left(w_{B i}\right)+18 \sum_{l=1}^{k} b_{l} \\
& +25 \log _{2}\left(w_{B i}\right)+3 \max _{l}\left(b_{i}\right)+66 \\
& \text { Power consumption } P_{B i}=\sum_{l=1}^{k} p_{l} \\
& \text { Test application time } T_{B i}=8 \times w_{B i} / f_{B i} \\
& \qquad\left(f_{B i}=f_{l}=f_{2}=\ldots=f_{k}\right)
\end{aligned}
$$

If the connection type of block $B_{j}=\left\{m_{1}, m_{2}, \ldots m_{k}\right\}$ is a serial connection,

$$
\begin{aligned}
& \text { Area } S_{B j}=0.75\left(\log _{2}\left(\sum_{l=1}^{k} w_{l}\right)\right)^{2}+2 k \log _{2}\left(\sum_{l=1}^{k} w_{i}\right) \\
& +25 \log _{2}\left(\sum_{l=1}^{k} w_{i}\right)+k\left(\log _{2} k\right)+9 b_{B i} k+14 b_{B i}+8 k+61
\end{aligned}
$$

$$
\left(b_{B i}=b_{1}=b_{2}=\ldots=b_{k}\right)
$$

Power consumption $P_{B j}=\max _{l}\left(p_{l}\right)$
Test application time $T_{B j}=8 \times\left(\sum_{l=1}^{k} w_{l}\right) / f_{B j}$

$$
\times \text { (number of background patterns) }
$$

The expressions for area calculation (7) and (10) do not consider the influence of timing conditions, but feedback is available from previous designs.

Parallel-connected memories are tested concurrently, and the power consumption is the sum of the power consumption of each memory. In contrast, serial-connected memories are activated one by one. Therefore, the power consumption is the maximum power consumption of the connected memories.

When a partition $\pi$ is found, the area, power consumption and test application time of each block are calculated using the above expression.

The total area of the memory BIST wrappers $S_{\text {total }}$ is calculated as the sum of $S_{B i}$.

$$
S_{\text {total }}=\sum_{i=1}^{k} S_{B i}
$$

To control each memory BIST wrapper, at least one BIST controller must be used. In this study, the number of memory BIST wrappers was reduced by using the proposed connections. There was therefore no increase in the number of controllers. In addition, our target design includes a lot of memories so that the area of the memory BIST wrappers is predominant. Therefore the area of the BIST controllers is disregarded.

To calculate the total test application time of a memory BIST under a power-consumption constraint, we used a rectangle packing algorithm that has been described elsewhere [5]. The algorithm optimizes the test schedule of each core so that the total test application time of an SoC is minimized under maximum power constraints. The inputs of the scheduling algorithm are the maximum allowed power consumption, the test application time, and the power consumption of each core. In this study, we considered a block to be a core. Therefore, we input $\left\{P_{B j}\right\}\left\{T_{B j}\right\}$ as the information for each core. In addition, we assumed the bit width of the inter-connect between each wrapper and control logic remained unchanged. We therefore disregarded the maximum TAM width.

To reduce the total area of memory BIST wrappers by memory BIST logic sharing, we formulated the following memory-grouping problem.

\section{Inputs:}

a) A set of memories $\mathrm{S}$ and

Information for each memory:

$\boldsymbol{M}=M_{\mathrm{i}}\left(b_{i}, w_{i}, p_{i}, f_{i}, x_{i} y_{i}\right)$

where, $b_{i}, w_{i}, p_{i}, f_{i}, x_{i}$ and $y_{i}$ are as follows:

$b_{i}$ : data bit width of $m_{i}$

$w_{i}$ : word depth of $m_{i}$

$p_{i}$ : maximum power consumption of testing $m_{i}$

$f_{i}$ : frequency of $m_{i}$

$x_{i}$ : X coordinate of $m_{i}$ 
$y_{i}:$ Y coordinate of $m_{i}$

Outputs:

a) A partition $\pi$ of a given set of memories $\mathrm{S}$ for which all the blocks satisfy the following conditions:

$G_{i p}$ is the graph induced on $G_{p}$ by block $B_{i}$.

$G_{i s}$ is the graph induced on $G_{s}$ by block $B_{i}$. $G_{i p}$ or $G_{i s}$ is a clique.

b) Type of connection of each block

c) Test schedule of each memory

\section{Constraints:}

a) Maximum distance of memory connection: $D$

b) Maximum available peak power of the SoC: $P$

c) Maximum test application time of memory: $T$

\section{Objective:}

To minimize $S_{\text {total }}$.

To solve this problem, an algorithm is proposed below.

\section{B. Memory Grouping Algorithm}

Fig.4 shows the pseudo code of the Memory Grouping Algorithm. Our proposed algorithm repeats division from 0 -partition that only one block includes all memory to obtaining a target partition. As the algorithm divides the block, $S_{\text {total }}$ increases. The min-cut method [2][3] is used to leave the possibility of the area reduction as much as possible. Moreover, it uses the following strategies to decide the compatibility of each block of the partition. Serial connection can reduce the area than parallel connection, and the power consumption is smaller than that of parallel connection. Therefore, it is possible that giving priority to serial connection reduces $S_{\text {total }}$. Based on this prospect, proposed algorithm searches for the partition that minimizes $S_{\text {total }}$ only using s-compatibility in the first search.

First, the algorithm initializes variables. The minimum value of $S_{\text {total }}$ is stored into $S_{\text {min }}$, and, in the first step, $S_{\text {min }}$ is set to the total area of memory BIST wrapper without sharing. The partition of a set of memory $\mathrm{S}$ is stored into $\pi$, and the initial partition is set to 0-partition of S. (line 1-2).

Next, the algorithm creates two compatibility graphs (line 3 ), and select s-compatibility graph as the graph $G$ that is used to find partition (line 4).

In order to check the compatibility of each block, the algorithm construct a set of graph $C_{\text {all }}$ (line 6). Each graph $G_{i}$ that is the member of $C_{a l l}$ is induced on $G$ by block $B_{i}$ that is the member of $\pi$.

Then, for all $B_{i}$ that include two or more memories, execute the following operations (line 7-21).

The minimum cut edge is calculated and delete them from $G_{i}$. By this operation, the vertex set $B_{i}$ is divided into two blocks, leaving much possibility of the area reduction. If all the graph of new graph set $C_{\text {all }}$ are clique, calculate $S_{\text {total }}$ and test schedule of the new partition $\pi_{\text {tmp }}$. If $S_{m i n}>S_{\text {total }}$ and the test scheduling succeeded, $\pi_{\text {tmp }}$ is stored into $\pi_{\text {best }}$ as the best partition, and $S_{\text {total }}$ is stored into $S_{\min }$ (line 8-17). If there is a graph $G_{i}$ that is not a clique, or the test scheduling failed, $\pi_{\text {tmp }}$ is stored into $\pi_{\text {next }}$ (line 18-20).
If there is no partition that should be tried, the first search is end (line22-24). Then the algorithm stores p-compatibility graph into $G$, and collects the blocks that have only one memory into one block (line25-29). Then, the algorithm searches for the partition that $S_{\text {total }}$ is minimized using p-compatibility (line5-24). In the second search, it doesn't touch the blocks in which two or more memories are included after first search. Their connection type is fixed to serial connection. The connection type of the rest is determined to be parallel connection.

This algorithm performs $\mathrm{n}(\mathrm{n}-1)$ times division and scheduling in the worst case. The complexity of the scheduling algorithm and min-cut algorithm are $O(V \log V)$ and $O\left(V^{2} \log V\right)$, respectively. Therefore the complexity of this algorithm is $O\left(V^{3} \log V\right)$.

In this paper, we described our method for a single port and the word access memory. However, this method is applicable to other memories if the compatibility is defined about the memory type and the connecting method, and the area, power consumption, test application time can be shown by expression.

\section{Experimental Results}

We carried out experiments to evaluate the proposed method. The proposed algorithm was implemented in $\mathrm{C}$ and the experiments were conducted on a $600-\mathrm{MHz}$ Windows PC. Table 1 shows the information in each memory used in the experiment. The 2-4th columns denote the data bit width, word depth, and operating frequencies, respectively. The 5th column shows the power consumption. In this experiment, the power consumption of each memory was a relative value in which memory No. 1 was assumed to be 100 under the following assumption:

a) The area is proportional to (number of words $\times$ number of bits).

b) The power consumption is proportional to the area.

c) The power consumption is proportional to the frequency.

Table1. Information on Memories

\begin{tabular}{|c|c|c|c|c|c|c|}
\hline \multirow[b]{2}{*}{ No. } & \multirow[b]{2}{*}{$\begin{array}{c}\text { \# data } \\
\text { bit } \\
\text { width }\end{array}$} & \multirow[b]{2}{*}{ \#Words } & \multirow[b]{2}{*}{$\begin{array}{c}\text { Frequency } \\
(\mathrm{MHz})\end{array}$} & \multirow[b]{2}{*}{$\begin{array}{c}\text { Power } \\
* 1\end{array}$} & \multicolumn{2}{|c|}{ Location } \\
\hline & & & & & $X$ & Y \\
\hline 1 & 16 & 128 & 133 & 100 & 10 & \\
\hline 2 & 16 & 128 & 133 & 100 & 20 & \\
\hline 3 & 16 & 128 & 266 & 200 & 30 & \\
\hline 4 & 16 & 128 & 266 & 200 & 40 & 10, \\
\hline 5 & 16 & 256 & 133 & 200 & 50 & \\
\hline 6 & 16 & 256 & 133 & 200 & 60 & 40 \\
\hline 7 & 16 & 256 & 133 & 200 & 70 & 50 \\
\hline 8 & 16 & 256 & 133 & 200 & 80 & \\
\hline 9 & 32 & 512 & 133 & 400 & 90 & \\
\hline 10 & 32 & 512 & 133 & 400 & 100 & \\
\hline
\end{tabular}

*1 Relative values in which memory No.1 is assumed to be 100 


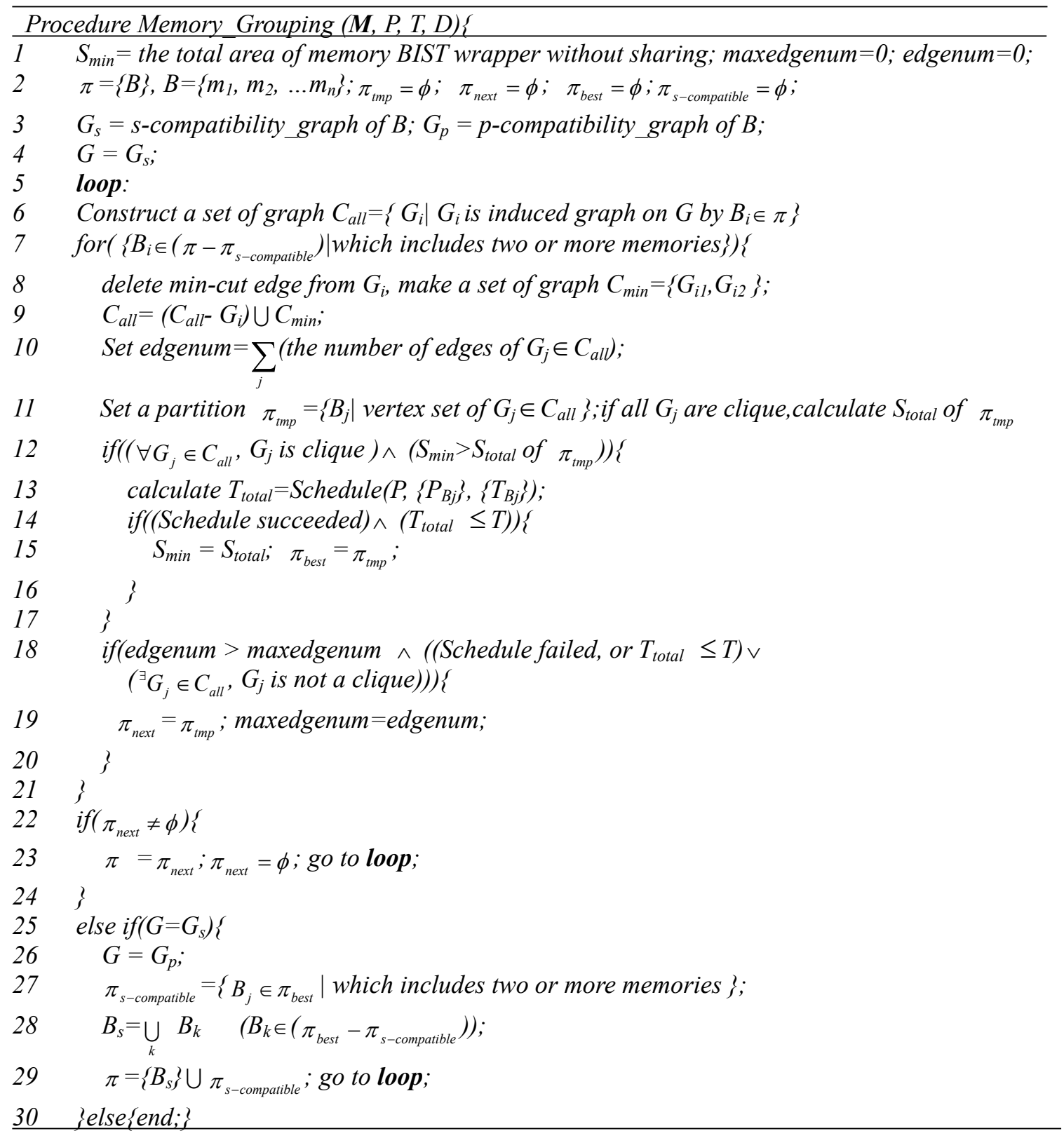

Fig.4 Memory Grouping Algorithm

The 6th and 7th columns show location. In this experiment, the number of memories was varied between 3 and 50 , and the program was executed respectively. When the number of memories was $\mathrm{N}<11$, we used No. 1 to $\mathrm{N}$, and for the rest, we extended the same set of No. 1-10, with the Y coordinate changing between 20 to 50 .

In an actual test, several background patterns (e.g. marching, checker, checker-bar) are used, but in this experiment, the test application time was calculated by assuming the number of background patterns $=1$. In addition, the following constraint values were used:

Maximum distance of memory connection: $\mathrm{D}=40$

Maximum available peak power of the SoC: $\mathrm{P}=5000$
Maximum test application time of memory:

$$
\mathrm{T}=300 \mu \mathrm{s}
$$

Experiments were carried out for the following five cases: (1) Not shared (all the memories had individual BIST wrappers); (2) parallel connection (memory BIST logic was shared using only parallel connection as described in the proposed technique); (3) serial connection (memory BIST logic was shared using only serial connection as described in the proposed technique); (4) parallel and serial connection (memory BIST logic was shared using both parallel and serial connection as described in the proposed technique); and (5) exhaustive search (memory BIST logic was shared using only parallel connection after an exhaustive search). Table 2 shows the experimental results. The first column 
shows the number of memories and the second column shows the total area of memory BIST wrappers without sharing. Columns 3-5 shows the total area of memory BIST wrappers using the proposed techniques. The third column shows the results of using only parallel connection, while the fourth column shows the results of using only serial connection. The fifth column shows the results of using both parallel and serial connection and the sixth column shows the minimum solution obtained using an exhaustive search.

We were only able to complete an exhaustive search when the number of memories was less than 7 . In these cases, the results of the exhaustive search showed that the memory BIST logic sharing technique reduced the area of the BIST wrappers by between 21.59 and $47.83 \%$ as minimum solutions. However, the technique achieved only $64.45 \%$ of the minimum solution in these cases, so there is room for improving the quality of the solution.

The average reduction ratio for parallel connection, serial connection, and parallel and serial connection were $21.08 \%$, $37.25 \%$, and $40.55 \%$, respectively. In all cases, parallel and serial connection achieved the best solution. This result demonstrates that selection from two types of connection methods reduces the area more than using a single connection method.

Finally, Figure 5 shows the execution time of the implemented memory-grouping program. In all cases, the program was executed within 10 seconds using the proposed algorithm. The technique thus obtained good results within a very short CPU time so it is suitable for practical application.

Table2. Area of Memory-BIST Logic

\begin{tabular}{|r|r|r|r|r|r|}
\hline \multirow{2}{*}{ \#mem } & \multirow{2}{*}{$\begin{array}{c}\text { not } \\
\text { shared }\end{array}$} & \multicolumn{3}{|c|}{ Proposed algorithm } & \multirow{2}{*}{ exhaustive } \\
\cline { 3 - 5 } & & P only & S only & S\&P & \\
\hline 3 & 2289 & 1967 & 1660 & 1660 & 1660 \\
\hline 4 & 2913 & 2591 & 2284 & 2284 & 2284 \\
\hline 5 & 3537 & 2893 & 2279 & 2279 & 2279 \\
\hline 6 & 4203 & 3559 & 3044 & 2722 & 2415 \\
\hline 7 & 4869 & 3863 & 3690 & 3368 & 2540 \\
\hline 8 & 5535 & 4529 & 3719 & 3397 & \\
\hline 9 & 6201 & 4793 & 3828 & 3506 \\
\hline 10 & 7242 & 5427 & 3122 & 3122 \\
\hline 11 & 8283 & 6021 & 6447 & 5678 \\
\hline 12 & 8907 & 7539 & 4703 & 4703 \\
\hline 13 & 9531 & 7394 & 5411 & 5089 \\
\hline 14 & 10155 & 7696 & 5406 & 5406 \\
\hline 15 & 10779 & 7998 & 5401 & 5401 & \\
\hline 20 & 14484 & 10854 & 6769 & 6769 \\
\hline 30 & 21726 & 16281 & 10455 & 10455 \\
\hline 40 & 28968 & 22070 & 23784 & 19662 \\
\hline 50 & 36210 & 27497 & 22964 & 21551 & \\
\hline
\end{tabular}

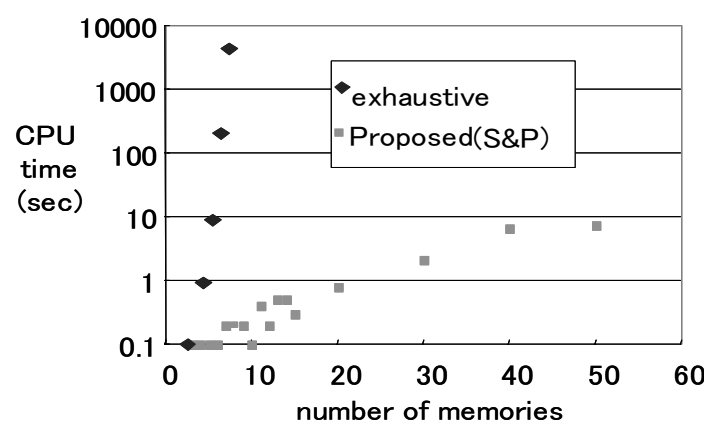

Fig.5 CPU Time of Memory Grouping program

\section{Summary and Conclusions}

A memory grouping problem was formulated and an algorithm to solve the problem was proposed. Experimental results showed that the proposed method reduced the area of memory BIST wrappers by up to $40.55 \%$. It was also shown that the ability to select from two types of connection methods reduced the area more than using a single connection method.

In future work we will investigate improving the quality of the solution and minimizing the test application time.

\section{Acknowledgements}

Authors would like to thank Prof. Michiko Inoue and Prof. Satoshi Ohtake and members of Computer Design and Test Lab. (Nara Institute of Science and Technology) for their valuable discussions.

\section{References}

[1] A.Benso, S.Di Carlo, G. Di Natale and P. Prinetto, "A Programmable BIST Architecture for Clusters of Multiple-Port SRAMs," in Proc. International Test Conf., pp557-566, October 2000.

[2] H. Nagamochi and T. Ibaraki, "A linear-time algorithm for finding a sparse k-connected spanning subgraph of a k-connected graph,” Algorithmica, vol. 7, 1992, pp. 583--596.

[3] H. Nagamochi and T. Ibaraki, "Computing the edge-connectivity of multigraphs and capacitated graphs, “SIAM J. Discrete Mathematics, vol. 5, 1992, pp. 54--66.

[4]Charles E. Stroud, A Designer's Guide to Built-In Self-Test, Kluwer Academic Publishers, The Netherlands, 2002.

[5]V. Iyengar, K. Chakrabarty and E. J. Marinissen, "On using rectangle packaging for SOC wrapper/TAM co-optimization," in Proc. VLSI Test Symposium, pp. 253-258, May 2002.

[6]Y. Huang, N. Mukherjee, S. Reddy, C. Tsai, W. Cheng, O. Samman, P. Reuter, and Y. Zaidan, "Optimal Core Wrapper Width Selection and SOC Test Scheduling Based On 3-Dimensional Bin Packing Algorithm," in Proc. International Test Conf., pp. 74-82, October 2002. 